\title{
EDITORIAL
}

\section{Glimmers of hope for functional dyspepsia}

F unctional dyspepsia is one of the most common gastrointestinal disorders encountered in clinical practice. Despite the frequency with which it occurs, this disorder remains poorly understood, and successful treatment options are limited. But, there are glimmers of hope. This special focus issue from Nature Reviews Gastroenterology \& Hepatology contains seven specially commissioned Reviews covering a broad spectrum of topics within the field of functional dyspepsia. Internationally renowned authors provide state-ofthe-art overviews of the progress being made today in understanding and treating this condition.

In 2006, the Rome III committee outlined diagnostic criteria for functional dyspepsia, and separated this disorder into two subgroups: epigastric pain syndrome and postprandial distress syndrome. The validity of this separation has been the subject of scrutiny ever since. In their Review, Jan Tack and Nicholas Talley describe the burgeoning number of epidemiological and pathophysiological studies investigating these subgroups. They suggest that the evidence is accumulating in favour of the division, but warn that further randomized controlled trials are urgently needed to clarify the situation.

One of the difficulties for developing effective treatments for functional dyspepsia is the underlying heterogeneity of the condition, which hints that multiple pathophysiological pathways have a role in symptom development. Hanne Vanheel and Ricard Farré describe changes in gastrointestinal tract function and structure, providing an integrated overview of how these changes might have a role in functional dyspepsia.

Despite these advances in understanding, we mostly remain in the dark regarding specific underlying factors that might lead to functional dyspepsia. However, progress is being made-experts are bringing to light ideas that could eventually improve the management of this condition. Particular foods, such as fatty foods, milk and dairy products, citrus fruits and spicy foods, have been implicated in triggering symptoms, and various mechanisms could explain these effects. Christine Feinle-Bisset and Fernando Azpiroz review the evidence regarding dietary and lifestyle factors in functional dyspepsia and outline a number of options that could be used as starting points for meaningful, large-scale studies in the future.

Continuing on the theme of potential causative factors, Lukas Van Oudenhove and Qasim Aziz discuss the role that psychosocial factors and psychiatric disorders might have in functional dyspepsia. Epidemiological studies have demonstrated an association between functional dyspepsia and psychopathology, and pathophysiological evidence could explain how these factors

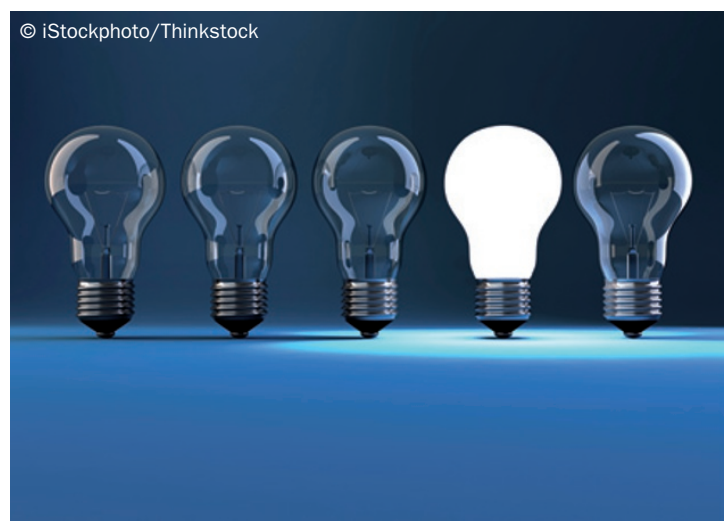

exert their role. Perhaps a biopsychosocial approach is warranted for understanding functional dyspepsia?

Helicobacter pylori infection is also postulated to be a potential cause of functional dyspepsia. As a causative agent in chronic gastritis, it is not hard to believe that this bacterium might also drive the symptoms of functional dyspepsia in some patients. Hidekazu Suzuki and Paul Moayyedi review the evidence for the role of $H$. pylori infection in functional dyspepsia, as well as providing critical analysis of the conflicting evidence regarding eradication of this pathogen as a means of management.

Not only are the causes of functional dyspepsia varied and poorly understood, but diagnosis can also be tricky. GERD is the most common upper gastrointestinal disorder, and the symptoms of these two conditions frequently overlap, leading to confusion for patients and clinicians alike. Eamonn Quigley and Brian Lacy shed some light on distinguishing between the two entities, particularly in relation to treatment options. However, once again, the need for further well-designed studies in precisely defined populations of patients is highlighted.

As mentioned, one of the key problems in functional dyspepsia is the lack of efficacious treatments-although acotiamide is a promising new agent for patients with postprandial distress syndrome. In the final Review of the issue, Michael Camilleri and Vincenzo Stanghellini discuss the important topic of current and emerging treatment options for this disorder.

Overall, therefore, exciting steps forward are being taken in the field of functional dyspepsia. As further trials are conducted and the pathogenesis of this disorder becomes clearer, the rather hazy picture regarding diagnosis and treatment should also begin to resolve. Thus, the evidence so far suggests that patients with functional dyspepsia can look forward to a brighter future.

doi:10.1038/nrgastro.2013.25

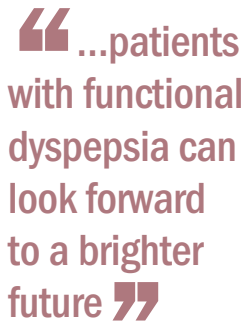

Isobel Leake is a Senior Editor of Nature Reviews Gastroenterology \& Hepatology.

Competing interests The author declares no competing interests. 